\title{
Epidemiología genética sobre las teorías causales y la patogénesis de la diabetes mellitus tipo 2
}

\author{
Carlos Jonnathan Castro-Juárez', Sergio Alberto Ramírez-García', Nemesio Villa-Ruano \\ y Diana García-Cruz ${ }^{2}$ \\ ${ }^{1}$ Instituto de Investigaciones Sobre la Salud Pública, Universidad de la Sierra Sur, Miahuatlán de Porfirio Díaz, Oax.; ${ }^{2}$ Instituto de Genética Humana \\ Dr. Enrique Corona Rivera, Departamento de Biología Molecular y Genómica, Centro Universitario de Ciencias de la Salud, Universidad de \\ Guadalajara, Guadalajara, Jal. México
}

\begin{abstract}
Resumen
La diabetes mellitus tipo2 (DM2) es un problema de salud pública mundial. Su etiología es multifactorial y se caracteriza por una gran heterogeneidad de alteraciones metabólicas. Las más frecuentes son la insuficiente producción de insulina, la resistencia a la insulina y el deterioro en el sistema de incretinas. El especialista debe comprender la naturaleza multicausal de la DM2en la era posgenómica. Esta naturaleza es determinada por el efecto aditivo de genes, así como del medio ambiente, y por ello no existe un modelo genético epidemiológico simple que explique su patrón de herencia. De aquí la necesidad de establecer la proporción de enfermedad que es determinada por los genes y la contribución de los factores ambientales, cuya combinación regula el umbral o nivel de tolerancia para que se desarrolle la diabetes. Dada esta complejidad de la DM2, en este trabajo se analizan las diversas teorías existentes sobre la causalidad de esta enfermedad, lo cual permitirá comprender la interacción del ambiente con el genoma humano y también conocer cómo los factores de riesgo o la predisposición a esta enfermedad influyen, sentando los fundamentos que delimitan la interacción del ambiente con el genoma.
\end{abstract}

PALABRAS CLAVE: Ambiente. Diabetes mellitus tipo 2. Genoma. Patogénesis. Salud pública.

\begin{abstract}
Diabetes mellitus type 2 (DM2) is a worldwide public health problem. The etiology of the disease is multifactorial and is characterized by great heterogeneity of metabolic disorders. The most common are the insufficient production of insulin, insulin resistance and impaired incretin system. The specialist must understand the multi-causal nature of DM2 in the post-genomic era. This nature is determined by the additive effect of genes and environment, so there is no simple genetic epidemiological model to explain the inheritance pattern. Hence the need to establish the proportion of disease that is determined by genes and the contribution of environmental factors, the combination of which regulates the threshold or tolerance level for diabetes development. Given this complexity in DM2 in this work are discussed the various existing theories of causality of this disease, which will permit us to understand the interaction between the environment and the human genome, and also to know how risk factors or predisposition to this disease influence, laying the grounds that delimit environment interaction with the genome.
\end{abstract}

KEY WORDS: Environment. Diabetes mellitus type 2. Genome. Pathogenesis. Public health

\author{
Correspondencia: \\ Diana García-Cruz \\ Sierra Mojada, 950, Edificio P, Nivel 2 \\ Col. Independencia \\ C.P. 44340, Guadalajara, Jal., México \\ E-mail: dianagarcr@ hotmail.com
}

Fecha de recepción: 05-12-2016

Fecha de aceptación: 23-03-2017

DOI://dx.doi.org/10.24875/GMM.17003064
Gac Med Mex. 2017;153:864-874

Contents available at PubMed www.gacetamedicademexico.com 


\section{Introducción}

La incidencia y la prevalencia de la diabetes mellitus tipo 2 (DM2) se ha reportado en hasta un $90 \%$ de los casos de totales de diabetes ${ }^{1,2}$. La Organización Mundial de la Salud ha predicho que para el año 2025 habrá más de 300 millones de diabéticos en el mundo, y de estos, 40 millones corresponderán a América Latina y el Caribe. La mitad no serán diagnosticados antes de que inicien las complicaciones crónicas ${ }^{3,4}$. Hay algunas poblaciones indígenas en que la prevalencia de DM2 es menor, debido a factores ambientales como la dieta (endémica/tradicional), que funciona como un factor protector ${ }^{5}$, pero existe un proceso de falta de culturización a la alimentación urbana, que aumenta considerablemente la prevalencia en comunidades rurales indígenas ${ }^{6}$.

La DM2 es una enfermedad de etiología multifactorial caracterizada por una gran heterogeneidad de defectos moleculares, como la insuficiente producción de insulina por las células $\beta$ del páncreas, la resistencia a la insulina y los defectos en el sistema de incretinas, que se traducen en alteraciones en el metabolismo de las grasas y de las proteínas ${ }^{4,7-10}$. Puede presentar sobreposición con la diabetes del joven maduro, producida por defectos genéticos en las células $\beta$ del páncreas, o bien pueden verse sobrepuestas endocrinopatías, infecciones, diabetes mellitus gestacional y formas de diabetes autoinmunitarias ${ }^{8}$. Forma parte de la evolución natural de los síndromes de Down, Klinefelter y Turner, entre otros ${ }^{8,9}$.

Epidemiológicamente, la DM2 ocurre con mayor frecuencia en población adulta9,11. Sin embargo, hay reportes que muestran una edad de inicio temprana, entre los 18 y antes de los 40 años ${ }^{12,13}$. En su conjunto, estos trabajos son una evidencia de la edad de inicio variable. Se ha propuesto que la diabetes está relacionada con el déficit parcial y con la resistencia a la insulina, así como con la respuesta a los hipoglucemiantes orales ${ }^{9,11}$. Es un trastorno complejo por la asociación con grados variables de predisposición hereditaria y la participación de factores de riesgo ambientales, como estrés, sobrepeso, obesidad central o abdominal, sedentarismo, dieta hipercalórica, dieta rica en grasas saturadas e hidratos de carbono simples, y pobre en frutas y verduras ${ }^{3,4,9-10,12-18}$.

\section{Teorías de la causalidad de la diabetes mellitus tipo 2}

El análisis sobre la patogénesis de la DM2 da un panorama global de la interacción del ambiente con el genoma humano y los factores de riesgo o predisposición, lo cual deben considerar los salubristas al diseñar estudios epidemiológicos ${ }^{7,8}$. La heterogeneidad genética y molecular de la diabetes es un reflejo de la naturaleza multifactorial de la DM2, la cual impacta directamente en la evolución de las complicaciones crónicas y en la respuesta al tratamiento. Por ello, no existe un modelo genético simple que explique la agregación familiar. Se requieren estudios que establezcan el porcentaje de enfermedad que es determinada por los genes y la contribución de los factores ambientales ${ }^{19}$. El presente trabajo discute la mayoría de las teorías sobre el origen de la diabetes, desde los estudios ambientales en los años 1960 hasta los biomédicos moleculares en 2016 .

\section{Teoría del genotipo ahorrador (Thrifty Genotype)}

En 1962, J.R. Neel propuso la teoría del «genotipo ahorrador», la cual explica que la DM2 es un proceso de evolución de los genes del metabolismo. En esta se comenta que existe un genotipo ahorrador, el cual se tradujo en los pobladores del Paleolítico (cazadores/recolectores) en un sistema metabólico eficiente para asimilar los nutrientes y almacenarlos en forma de grasa en los periodos de abundancia de alimento, lo que garantizó su supervivencia en los periodos de hambruna prolongados durante la Cuarta Glaciación. En aquella sociedad primitiva vivieron los pobladores que tenían tal genotipo adaptativo. Los herederos actuales de estos pobladores son las sociedades poscoloniales latinoamericanas, que por la transición a sociedades industrializadas con alta ingesta de comidas energéticas (ingesta abundante) sufren una selección negativa que permite la aparición de obesidad y de diabetes. Neel citó que este proceso ocurrió en Europa en el siglo XVIII, lo cual produjo una nueva adaptación de las sociedades, donde solo sobrevivieron los individuos sin el genotipo ahorrador ${ }^{20}$. El genotipo ahorrador son muchos genes, y es una línea que se debe seguir en la epidemiología de la diabetes en Latinoamérica, ya que explica la diabetes en las sociedades poscoloniales, las cuales presentan abundante consumo de alimentos altamente energéticos. Muchos de estos genes codifican para factores de crecimiento, receptores para hormonas, reguladores vasculares y reguladores del metabolismo hepático de lípidos, entre otros. Antiguamente se había propuesto al gen $A P O E$ como parte de ese genotipo ${ }^{8}$. En la actualidad, en población mexicana hemos 
explorado los genes LRP2, HPSE y ELMO1. Se ha propuesto a HPSE, que codifica para la heparanasa, una enzima que regula el metabolismo del heparán sulfato de la matriz extracelular, importante en la unión de la apolipoproteína $\mathrm{E}$ al endotelio vascular, cuya alteración favorece la aterogénesis y la disfunción vascular asociada a la diabetes; $L R P 2$, que codifica para un receptor de las lipoproteínas, el cual funciona a través de un mecanismo de endocitosis renal, porque su desregulación conduce a dislipidemia y diabetes; y ELMO1, un regulador de la citocinesis en el centrosoma, cuya desregulación conduce a diabetes. Estos genes, por estudios de asociación, han mostrado su efecto en el riesgo para la diabetes de larga evolución, cuyo fenotipo clínico es la resistencia a la insulina. La interacción de estos genes pleiotrópicos en estudios de asociación con sus polimorfismos puede explicar el riesgo de desarrollo de hipertensión, dislipidemias, hígado graso, sobrepeso y obesidad, que son estadios previos a la $\mathrm{DM} 2^{21-24}$.

\section{Resistencia a la insulina o genotipo no tan ahorrador (not so thrifty gene)}

La información genética se fue moldeando lentamente, sin grandes cambios importantes, durante cada una de las etapas del proceso evolutivo desde el Ardipithe curamidus, de vida arbórea, hasta el Homo sapiens actual, determinando así nuestros requerimientos nutricionales. Durante estas etapas evolutivas surgió el "genotipo ahorrador», expresado en una resistencia a la leptina, el «genotipo no tan ahorrador», con resistencia a la insulina. La resistencia a la insulina muscular fue clave para sobrevivir en los periodos de hambruna, que eran muy frecuentes para el Homo ergaster. La gluconeogénesis hepática y renal permitió mantener la glucemia en valores normales durante el ayuno; esta adaptación se hace mucho más efectiva si el músculo esquelético presenta resistencia a la insulina, ya que este tejido se obliga a utilizar ácidos grasos provenientes de las reservas del tejido adiposo, reservando a su vez la glucosa para un consumo casi exclusivo por parte del cerebro y de los eritrocitos, los dos tejidos altamente dependientes de la glucosa para sus funciones metabólicas ${ }^{20}$. La resistencia a la insulina muscular, al producir altos concentraciones de insulina circulante, favorece la acumulación de triglicéridos en el tejido adiposo, con lo cual se favorece la adipogénesis ${ }^{25}$. Para explicar esta teoría se ha propuesto al gen CPN10, que codifica a la calpaína 10, una proteasa muscular; el genotipo heterocigoto del polimorfismo SNP43 de esta se relaciona con una menor cantidad de RNAm en el músculo estriado y proteólisis ${ }^{18}$.

La resistencia a la insulina fue el mecanismo que permitió a los migrantes de la Cuarta Glaciación sobrevivir durante los periodos de ayuno prolongado, conservando la masa muscular, lo que les permitió cazar con éxito en la primera oportunidad o poder escapar de los depredadores. Conservar la masa muscular significó aumentar la glucosuria y disminuir la proteólisis. Los estudios sobre el gen CPN10 apoyan esta teoría, ya que este codifica para una proteasa de cisterna citosólica muscular. En los indios Pima, los portadores homocigotos del alelo $\mathrm{G}$ del polimorfismo SNP49 presentan menor cantidad de ARNm en el músculo estriado. Las variaciones en este gen ya han sido asociadas con DM2 en población mexicana. Nosotros postulamos que las variantes del gen GLUT2 (que codifica para el transportador tipo 2 de la glucosa y está implicado en el síndrome de Fanconi renal, una glucogénesis hepatorrenal con glucosuria, fosfaturia, aminoaciduria y mucopolisacariduria) pudieran apoyar esta hipótesis. En un estudio exploratorio en pacientes con DM2 en población del sur de México no encontramos asociación de este gen, pero debe seguirse estudiado, ya que su producto, el transportador de tipo 2 de la glucosa, es uno de los blancos actuales en el tratamiento de la DM2, por ser glucosúrico².

\section{Teoría del fenotipo ahorrativo}

La hipótesis del fenotipo ahorrador plantea que la subnutrición, tanto intrauterina como infantil, puede programar una expresión génica para beneficiar la sobrevida. Se protege al cerebro frente a otros tejidos, lo que conduce a una programación metabólica condicionada por la escasez del sustrato bioenergético ${ }^{26,27}$. La malnutrición intrauterina, en particular el déficit proteico, ha demostrado una alteración en la función del páncreas con disminución de las células de los islotes de Langerhans y una menor capacidad secretora de insulina. De acuerdo con esta hipótesis, la subnutrición, que se caracteriza por un bajo peso al nacer, provocaría una programación in utero que explicaría la aparición de la mayor parte de la obesidad, el síndrome metabólico y la DM2 en un adulto que nació con bajo peso para la edad gestacional, y que posteriormente es expuesto a un ambiente de abundancia alimentaria. Aún más, las evidencias de 
numerosos estudios indican que esta programación no se limita a la primera generación $n^{18,27,28}$.

\section{Teoría de la programación (hipótesis de Barker)}

Esta teoría de asociación sustenta que si existe un estímulo o agresión in utero (malnutrición intrauterina) durante un periodo crítico, este produce un cambio temporal o permanente en la estructura y la función de un organismo (programación fetal) ${ }^{29,30}$. Actualmente se han identificado cuatro fenotipos al nacimiento relacionados con enfermedades en la edad adulta, de los cuales dos explican las alteraciones relacionadas con el control glucémico. El primero indica que recién nacidos delgados tienden a ser resistentes a la insulina cuando llegan a adultos, y el segundo correlaciona los lactantes de talla baja y peso elevado con una menor producción de insulina, generando DM2 en edades adultas. Esto da lugar a la hipótesis del origen fetal de la enfermedad, que originaría DM2 y enfermedades crónicas debido a la adaptación del feto a la malnutrición intrauterina ${ }^{31,32}$.

\section{Impronta genética}

El ser humano presenta una marca epigenética única que es parcialmente heredada y parcialmente generada in utero, con cambios en la vida adulta ${ }^{33,34}$. Estos cambios epigenéticos se generan por diversas vías, como la metilación de histonas y genes, la acetilación de histonas, la ubiquitinación, la sumoilación, la fosforilación, la ribosilación, la glucosilación y los RNA no codificantes. Se ha documentado que en la fase de la concepción se presentan dos momentos de apagado y programación de un nuevo gen: el primer momento se da durante la gametogénesis y el segundo en la preimplantación, cuando el genoma se desmetila; durante estos dos procesos de metilación, el programa epigenético se vuelve vulnerable a las alteraciones generadas por el medio ambiente fetal, existiendo teorías de que estas alteraciones generarían padecimientos en la edad adulta, como la $\mathrm{DM} 2^{35}$. Sin embargo, datos recientes indican que durante la infancia, la adolescencia y la pubertad también pueden ocurrir alteraciones epigenéticas que afectarían el sistema endocrino, ya que en estas etapas hay un aumento relativo de la síntesis de ADN y crecimiento celular ${ }^{36,37}$.

La alimentación brinda un papel protector o desencadenante de estos procesos. Actualmente se ha identificado que la disminución por parte de la dieta en el aporte de donadores de grupos metilo (folatos, metionina y colina), y el consumo de dietas pobres en azufre, producen una modificación en la formación de cisteína, asociándose a una disminución de la metilación del ADN. Sin embargo, a diferencia de los polimorfismos genéticos, que son permanentes, estas alteraciones ambientales no lo son; no obstante, los factores como el estrés, el poco ejercicio, la inadecuada dieta, los procesos inflamatorios, la hiperglucemia, la obesidad y el estrés oxidativo van a condicionar una sobreexpresión de algunos de los genes. Así mismo, algo importante de los errores en el proceso de metilación del ADN es que aumentan conforme se va envejeciendo, ocasionando un incremento en el riesgo de padecer $\mathrm{DM} 2^{38,39}$.

Los factores ambientales, tales como la hiperglucemia que generan los alimentos con un índice glucémico alto, contribuyen a producir compuestos tóxicos por cuatro vías fundamentales: la vía del poliol, la de formación de productos terminales de la glucosilación avanzada (PTGA), la vía de activación de la proteína cinasa $C$ y la vía de la hexosamina; esto ocasiona una producción mayor de especies reactivas de oxígeno (ERO), especies reactivas de nitrógeno y aumento de $\mathrm{O}_{2}^{-}$, generando una situación de estrés oxidativo que conduce a vías dirigidas a alteraciones epigenéticas y a la apoptosis ${ }^{40}$.

Diversos estudios explican que la hiperglucemia afecta la expresión de determinados genes y modificaciones en la cromatina, como es el caso de la asociación entre los cambios químicos de las colas amino-terminales de la histona $\mathrm{H} 3$ y la metiltransferasa Set7 específica de lisinas. Esta expresión ayuda a mejorar la accesibilidad de la cromatina y la transcripción de los genes de las células $\beta$, indicando que esta es la causante de la expresión génica vascular en respuesta a episodios anteriores de hiperglucemia ${ }^{40}$. Esto se apoya en los hallazgos en un modelo experimental de ovejas que sufrieron desnutrición materna moderada, en las cuales se encontraron reducidos la metilación de los promotores del gen (POMC) y el receptor de glucocorticoides en el hipotálamo fetal, alterando la regulación del balance energético y asociándose con una menor actividad de la ADN metiltransferasa y una alteración de la metilación, así como con acetilación de las histonas, generando la hipótesis de que las dietas hipocalóricas o bajas en proteínas durante el embarazo están implicadas en la programación de la descendencia hacia el desarrollo de obesidad y $\mathrm{DM}^{41}$. 
Otro estudio con gemelos monocigóticos de entre 53 y 80 años de edad, divergentes para la DM2, identificó 789 sitios CpG en el músculo esquelético y 1458 en el tejido adiposo subcutáneo diferencialmente metilados ${ }^{42}$, siendo que algunos de ellos se localizaron en genes tan importantes como PPARGC1A y HNF4A, lo que podría llevar a un aumento de la producción de glucosa hepática que, a su vez, contribuiría a aumentar el riesgo de hiperglucemia, y si se mantiene, a generar resistencia a la insulina y DM2. Volkmar, et al. ${ }^{43}$ describieron una cantidad considerable de genes hipermetilados en los islotes de pacientes con DM2 que participan en vías identificadas para mantener la supervivencia y el funcionamiento de las células $\beta$. Se sugiere que estos mecanismos epigenéticos están implicados en la disfunción de la célula $\beta$ y la génesis de la DM2.

Respecto al consumo de grasas, se ha reportado un patrón en la patogénesis de la DM2 que sugiere que dietas hiperlipídicas e hipercalóricas generan un aumento de la producción de ácidos grasos de cadena corta como subproductos de ácidos grasos de cadena larga, lo que conduciría a cambios en la metilación del ADN que causan la hipometilación del promotor y la desregulación de las citocinas proinflamatorias, generando una resistencia periférica a la insulina ${ }^{38}$. Estos resultados señalan que diversos mecanismos epigenéticos pueden estar implicados en la disfunción de las células $\beta$ y en la patogénesis de la diabetes, abriendo la puerta al estudio del papel de la nutrición, el estrés oxidativo y los procesos inflamatorios.

Actualmente se han identificado cuatro fenotipos al nacimiento asociados con enfermedades en la edad adulta, de los cuales dos explican las alteraciones relacionadas con el control glucémico. El primero indica que recién nacidos delgados tienden a ser resistentes a la insulina cuando llegan a adultos, y el segundo correlaciona los lactantes de talla baja y peso elevado con una menor producción de insulina, generando DM2 en edades adultas. Esta teoría, un poco olvidada, está estrechamente relacionada con la impronta genética. La programación metabólica para la vida adulta se realiza en el último trimestre del embarazo, y está asociada con la resistencia a la insulina. Uno de los genes que pudiera estar implicado es el gen que codifica para la aromatasa, CYP19A1, ya que convierte los estrógenos en andrógenos en los tejidos periféricos, como el tejido graso y el músculo, entre otros. Se asocia con un fenotipo clínico caracterizado por virilización, hiperandrogenismo, diabetes gestacional y resistencia a la insulina, la cual es un mecanismo fisiopatogénico de la reprogramación metabólica. Algunos estudios preliminares epidemiológicos, en los que hemos colaborado, en población mexicana respecto a este gen, muestran su asociación con la DM2 y la retinopatía diabética.

Otro línea por explorar, que requiere más estudios, es el alto consumo de grasas saturadas. Se ha sugerido que dietas hiperlipídicas e hipercalóricas generan un aumento de la producción de ácidos grasos de cadena corta como subproductos de ácidos grasos de cadena larga, lo que conduciría a cambios en la metilación del ADN que causan la hipometilación del promotor y la desregulación de las citocinas proinflamatorias, generando una resistencia periférica a la insulina, lo cual puede asociarse con la DM2 que cursa con disfunción de las células $\beta$. Uno de los genes que hemos explorado en este sentido en población mexicana es el gen TJP1, que codifica para la unión estrecha tipo 1, la cual participa en los contactos célula-célula y es importante en la morfogénesis pancreática durante la embriogénesis; también regula en las células el flujo de nutrientes a nivel apical y basolateral, como la glucosa y el colesterol ${ }^{44}$.

\section{Teoría de las comidas genéticamente desconocidas}

El consumo de comidas para las que no está diseñado genéticamente el humano puede ser un factor que conduce al desarrollo de DM2. Esta hipótesis se ha puesto de moda en los últimos 20 años. Las evidencias en este aspecto pueden ser aplicadas primeramente al consumo de azúcares refinados en los edulcorantes (contenidos en las bebidas gaseosas), que conducen al desarrollo de hipertrigliceridemia, un factor de riesgo para el desarrollo de DM2. Por otra parte, existe un cambio en el tipo de consumo de sales contenidas en los alimentos: el humano metabólicamente está diseñado para el citrato de potasio, pero muchos alimentos actualmente tienen un alto contenido de cloruro de sodio, una sal con la cual hemos coevolucionado, pero que puede inducir nefroesclerosis y favorecer el daño renal en los diabéticos, como se ha demostrado en modelos múridos. Sin embargo, el citrato de potasio es nefroprotector y cuida la salud renal ${ }^{8}$. Esto es polémico, ya que los humanos han coevolucionado con el cloruro de sodio, pero también explica la alta frecuencia de hipertensión arterial sistémica muy asociada con la diabetes, 
lo cual nos dice que hay factores de riesgo del ambiente, como la dieta rica en cloruro de sodio, que favorecen el desarrollo de complicaciones vasculares en los diabéticos.

A pesar de las polémicas actuales en torno a los alimentos hechos a base de organismos genéticamente modificados, mismos que podrían ser considerados como genéticamente desconocidos, aún quedan muchas incógnitas sobre el alcance del concepto y sus efectos reales. Los mecanismos de transferencia horizontal de genes no solo se limitan a microorganismos, sino a también a plantas, siendo por tanto un proceso de transgénesis «natural». Como ejemplo se puede citar el camote o papa dulce (Ipomoea batatas [L.] Lam.), un alimento tradicionalmente consumido por siglos en algunos países del continente americano, que ha demostrado guardar una estrecha relación de intercambio de genes con Agrobacterium tumefaciens (bacteria usada para generar vegetales transgénicos) durante miles de $a$ ños ${ }^{45}$. A pesar de este hecho, el camote es y será considerado inocuo por las poblaciones que lo consumen y por los estudios científicos que revelan sus beneficios, así como sus riesgos conocidos, para la salud. En contraste, los estudios sobre el efecto nocivo de los transgénicos, en particular del maíz transgénico consumido por generaciones, no han sido fructíferos ni contundentes, ya que no existe información actual en la literatura científica que demuestre su asociación con el desarrollo de la DM2. Al contrario, se han observado una pérdida de la objetividad, inconsistencias y posibles manipulaciones que los vinculan al desarrollo de enfermedades consideradas problemas de salud pública mundiales ${ }^{46}$. Este hecho podría estar asociado a conflictos de intereses puramente comerciales y no científicos.

Por otra parte, hay que considerar que el humano está diseñado para una tasa metabólica basal de 4.18 MJ/l (el límite fisiológico impuesto por la evolución), y al consumir en exceso comidas ricas en grasa y sacarosa en estado sólido (dieta occidental) se favorece la génesis de la DM2 debido a la alteración del balance energético. Las poblaciones que todavía tienen el genotipo ahorrador, como los indios Pima, son metabólicamente incapaces de hacer frente a estos hábitos dietéticos, que son diabetogénicos para ellos, mientras que otras poblaciones, como los europeos, han logrado adaptarse ${ }^{47}$. Los indios Pima, así como otras poblaciones, se moldearon metabólicamente por un entorno nutricional de baja densidad grasa y energética (hace miles de años, durante la Cuarta
Glaciación), donde las dietas que contienen > 10-15\% de grasa eran imposibles obtener ${ }^{47}$. Un ejemplo es el hecho de que la mortalidad por enfermedad coronaria es 16.7 veces mayor en los EE.UU. que en la China rural, donde la ingesta de grasa es menor y la concentración media de colesterol es de $3.28 \mathrm{mmol} / \mathrm{l}$ (127 mg/dl), en comparación con $5.24 \mathrm{mmol} / \mathrm{l}$ (203 mg/ dl). Se ha reportado incluso que los sujetos europeos, a pesar de su adaptación relativa, son incapaces de mantener el balance energético (aumentan de peso) al cambiar de una dieta de baja densidad energética a una dieta de alta densidad energética ${ }^{48}$. Esto no sorprende si tenemos en cuenta que, cuando las poblaciones eran cazadoras-recolectoras, las dietas ricas en grasas eran prácticamente inexistentes, y por lo tanto tales dietas solo pueden verse en la nutrición actual.

No obstante, se desconoce parcialmente el efecto que pudieran generar algunos compuestos contenidos en las plantas. En la actualidad, en ciertas poblaciones de descendencia indígena, como la población del sur de México, se observa que algunos alimentos vegetales, principalmente en forma de extractos 0 infusiones, logran disminuir los cuadros de hiperglucemia y llegan en algún momento a considerarse como parte del tratamiento de la diabetes mellitus. A partir de ensayos in vitro o in vivo con modelos animales se ha logrado identificar que la actividad antioxidante e inhibitoria (compuestos fenólicos y terpenos) de enzimas del metabolismo de los hidratos de carbono que presentan estos alimentos sustenta su uso tradicional, o bien a través de la química hipoglucemiante de productos naturales ${ }^{49}$, lo que supondría que el organismo humano ha evolucionado junto al consumo de estos, principalmente en las poblaciones que lo usan como tratamiento tradicional.

\section{Hipótesis del origen isquémico}

Por otra parte, se ha reportado una patogénesis diferente, la cual explica que la causa primaria de la DM2 es de origen isquémico por una disminución transitoria o permanente del riego sanguíneo en los núcleos hipotalámicos anteriores, provocando hiperexcitación de las vías parasimpáticas descendentes y del eje hipotálamo anterior, incrementando las concentraciones sanguíneas de glucosa y de lípidos, así como acumulación de tejido adiposo, siendo este último el principal implicado en la resistencia a la insulina y la hiperinsulinemia. La isquemia provoca una deficiencia crónica progresiva en la secreción de 
insulina, lo que favorece la necrosis de las células $\beta$ por falta de irrigación. En este sentido, se ha demostrado que los islotes tienen alta dependencia del aporte sanguíneo, lo cual los hace muy vulnerables a los cambios isquémicos ${ }^{50}$.

\section{Síndrome de resistencia orgánica múltiple y modelo de Corkey}

Se ha reportado que existe, tanto en el músculo cardiaco como en el esquelético, el endotelio, el hepatocito, el adipocito y la célula $\beta$, una resistencia múltiple y simultánea a nivel de receptor para la leptina, la adiponectina y la insulina, la cual conduce a hiperleptinemia, hiperinsulinemia y adiponectinemia, que se traducen en la coexistencia de obesidad, hiperfagia y $\mathrm{DM} 2^{51}$. Es controvertido si la hiperinsulinemia es causa o consecuencia de la obesidad y la DM2. En este sentido, Corkey ${ }^{52}$ observó una excesiva respuesta secretora de las células $\beta$, posiblemente debida a factores ambientales o factor $\mathrm{X}$ (ficticio), lo que conduce a hiperinsulinemia y posteriormente a resistencia a la insulina. También esta secreción puede estar determinada genéticamente, y así, los factores modifican indirectamente los valores circulantes de indicadores redox, con un incremento de la secreción de insulina, la cual es mantenida, así como las señales generadas en el organismo, causando resistencia a la insulina de origen hepático e incremento de la masa corporal, y posteriormente el desarrollo de $\mathrm{DM} 2^{52}$. Uno de los genes que vale la pena explorar en el sentido de la hipótesis es ATXN2, ya que tiene muchos efectos pleiotrópicos simultáneos en diferentes tejidos y su disfunción en modelos múridos produce hígado graso, resistencia a la insulina, obesidad y problemas neurológicos. Algunos polimorfismos están asociados con obesidad; nosotros hemos encontrado en México la asociación con diabetes de VNTR (Variable Number of Tandem Repeats) de este gen en pacientes cuyo fenotipo clínico es la resistencia a la insulina y obesidad.

\section{La enfermedad conformacional y su relación con el envejecimiento y el estrés oxidativo}

Las enfermedades conformacionales surgen de la agregación de proteínas disfuncionales en conformaciones no nativas. La DM2 puede definirse como una enfermedad conformacional porque una proteína de las células $\beta$ constituyente, polipéptido amiloide de los islotes, sufre un cambio en la estructura terciaria seguido por la autoasociación y la deposición de teji$\mathrm{do}^{53}$. Por un lado, la DM2 se asocia con varios trastornos metabólicos que dan como resultado la producción excesiva de ERO y estrés oxidativo. Estas ERO ponen en marcha una serie de reacciones redox que puede resultar en nitrógeno inestable y tiol que contribuyen al estrés redox adicional ${ }^{53}$. La capacidad de una célula para hacer frente a las ERO y el estrés oxidativo requiere chaperonas funcionales, la producción de antioxidantes, la degradación de la proteína y una cascada de eventos intracelulares conocidos colectivamente como respuesta de las proteínas desplegadas. Se sabe que las células $\beta$ son particularmente susceptibles a perturbaciones en este sistema de control de calidad, y que las ERO desempeñan un papel importante en el desarrollo o la progresión de la $\mathrm{DM} 2^{53}$. El estrés oxidativo y la producción de insulina contribuyen al aumento de estrés del retículo endoplásmico, al mal plegamiento de las proteínas y a la inducción de la respuesta de las proteínas desplegadas. Como el sistema de la célula de control de calidad se abruma, se producen cambios conformacionales de los islotes intermedios polipéptido-amiloide y la generación de oligómeros estables con un antiparalelo estructura cruzada de lámina $\beta$ plegada, que eventualmente se acumulan como lesiones ocupantes de espacio dentro de los islotes ${ }^{53}$.

En principio se puede decir que las disfunciones de diferentes órganos, como el páncreas, están dadas por la disminución de las células, hecho que Hayflick ${ }^{54}$ documentó al observar que los fibroblastos humanos tenían una capacidad limitada de replicación in vitro; sin embargo, diversos estudios rechazan que el comportamiento en cuanto al proceso de envejecimiento celular no pueda ser replicado en animales enteros (sobre todo en vertebrados superiores) ${ }^{55}$.

Se ha reportado a la DM2 como una enfermedad conformacional, asociada a la transición estructural de las proteínas fisiológicas con estados intermedios o de proteínas patológicas. Es posible que la naturaleza implacable de la progresión de esta enfermedad se pueda entender en relación con el acúmulo progresivo de estas proteínas aberrantes o desconocidas para el organismo, como puede apreciarse en otras enfermedades conformacionales como el Alzheimer, las producidas por priones o el Parkinson, entre otras. Sin embargo, al hablar de la relación del envejecimiento con los procesos patológicos mencionados y los propios relacionados con una disminución de los 
procesos biológicos, como el metabolismo de los isómeros estructurales, resulta difícil, de diversas formas, dentro de estas poder separar el efecto que tienen la adaptabilidad y la resistencia al estrés, así como la disminución del organismo para poder mantener las funciones biológicas en equilibrio. Varios autores manejan diversas clasificaciones en cuanto a las teorías que producen el envejecimiento. En este manuscrito se han abarcado las que podrían darnos una explicación de la génesis en cuanto al proceso de disminución de la producción de insulina por las células $\beta$ del páncreas. En población mexicana, precisamente en diabéticos tipo 2 con disfunción de las células $\beta$ y defecto en la secreción de insulina, analizamos el efecto del polimorfismo p.A1334 del gen TJP1, que produce un cambio conformacional en la unión estrecha tipo1, la cual es muy importante para la morfogénesis pancreática en el periodo embrionario, que mantiene el porcentaje y la función de las células $\beta$ en el adulto $^{56}$.

\section{Teorías exógenas}

Dentro de las teorías que sustentan la disminución del funcionamiento de las células $\beta$ está la de la acumulación de los productos terminales de PTGA. En la DM2 no controlada existe un incremento en la cantidad de PTGA ${ }^{57}$ (fenómeno que Strehler ${ }^{58}$ llamó progresivo). Debido al incremento de PTGA se modifica la elasticidad de las arterias, con disminución de la solubilidad del colágeno y aumento en la anchura de las membranas basales retinianas, estableciéndose entrecruzamientos de polímeros como meloidinas (generados por la fructosamina) y cadenas proteicas ${ }^{57}$.

La teoría del estrés oxidativo, en la que son protagonistas los radicales libres, propone que existe una progresiva destrucción de las células $\beta$ secundaria a los procesos de oxidación y metabolismo que generan los radicales anión, el radical hidroxilo, el superóxido $\left(\mathrm{O}_{2}^{-}\right)$y el peróxido de hidrogeno $\left(\mathrm{H}_{2} \mathrm{O}_{2}\right)^{57}$. Estos forman parte de los niveles de ERO, siendo la mitocondria sensible a su acumulación ${ }^{59}$, por lo que disminuirá la producción de insulina, originando un incremento en la glucosa posprandial en un inicio y aumentando los PTGA ${ }^{60,61}$. Sin embargo, actualmente la teoría de la acumulación de ERO es discutible, ya que en Caenorhabditiselegans se ha demostrado que el principal determinante en la expectativa de vida y la longevidad son los valores de la superóxido dismutasa 4 dependiente de Cu/ZnS extracelular ${ }^{62,63}$. Nosotros hemos propuesto en este sentido al gen CSB, el cual participa en el sistema de reparación del ADN acoplado a la transcripción. Su disfunción produce una enfermedad conocida como síndrome de Cockayne (enfermedad progeroide), el cual cursa con diabetes mellitus; esto puede estar relacionado con la deficiencia secundaria de melatonina por la calcificación de la glándula pineal, sobre todo porque la melatonina es un potente antioxidante. Sin duda, es un factor que reduce la longevidad y aumenta el estrés oxidativo. Todavía lo seguimos explorando en diabetes del adulto intermedio y del adulto mayor en México ${ }^{64}$.

También se ha manejado en estudios recientes la hormesis, refiriéndose a un efecto bifásico o adaptativo por el que concentraciones bajas de ciertas moléculas toxicas tienen efectos positivos sobre la expresión de genes de respuesta a las ERO, y dosis altas son inhibitorias, sustentando que cierto nivel de estrés oxidativo es necesario para prolongar la expectativa de vida ${ }^{63}$.

\section{Teoría génica y mixta}

Esta teoría propone que el envejecimiento se debe a factores relacionados con el genoma de la célula; hay más de 100 genes que están íntimamente relacionados con la longevidad. Se ha sugerido que el envejecimiento se produce por alteraciones en el ADN o en la transcripción a mRNA, o en la traducción a proteínas, indicando que es un proceso programado en el genoma desde el nacimiento y que se ve afectado por exposiciones a agentes externos ${ }^{55,65,66}$.

Se ha sugerido que existe relación entre el envejecimiento celular y la inestabilidad en el genoma mitocondrial por efecto en la disminución en la reparación mitocondrial a causa de las ERO, lo que generaría incapacidad para sintetizar ATP (compuesto importante para la generación de insulina, ya que es una síntesis dependiente de energía), resultando en la senescencia del funcionamiento y desencadenando en años siguientes la $\mathrm{DM}^{67}$. Este hecho lo demostraron Fleming et. al. ${ }^{68}$ con su hipótesis de la mutagénesis mitocondrial intrínseca, reportando daño en el ADNmt y conduciendo a una síntesis de proteínas inadecuadas en la membrana interna; esto se debe a que carece de histonas y de mecanismos de reparación apropiados. Sin embargo, diversos autores indican que se han identificado factores de reparación mitocondriales, entre ellos la vía de reparación por escisión de base; aunque trabajos recientes han demostrado que no es tan eficiente en las mitocondrias de los mamíferos, también hay indicios de la presencia de 
procesos de reparación más complejos ${ }^{68-70}$. Por lo tanto, la comprensión de los procesos de reparación del ADNmt de los mamíferos es un reto actual y es probable que conduzca al esclarecimiento de la etiología de las mutaciones y deleciones comunes, que se cree que son las causantes de varios trastornos humanos, como la DM2 y el fenotipo de envejecimiento.

\section{Teoría de la acumulación de productos de desecho}

Estos se generan en el proceso de envejecimiento celular y se originan de la rotura de productos citoplásmicos perjudiciales para la célula, lo que ocasiona que las células puedan evitar su mortalidad, creciendo y diluyendo estos productos de desecho. Sheldrake ${ }^{71}$ propuso a la lipofuscina como un producto perjudicial. Respecto al compuesto que ha mantenido diversos debates sobre si es citotóxico para la célula $\beta$, este es el péptido amiloide o amilina. Se ha debatido extensamente si la presencia de depósitos de sustancia amiloide puede estar involucrada en la patogenia de la DM2, o si simplemente se trata de la consecuencia de la enfermedad. No obstante, el hecho de que tales depósitos en pacientes con DM2 se asocien a una reducción en la cantidad de células $\beta$ sugiere que pueden constituir un importante factor etiopatogénico en el desarrollo de la insuficiente producción de insulina ${ }^{71,72}$.

El péptido amiloide transita en el plasma en concentraciones mínimas (picomolares), siendo que las cantidades varían de individuos no diabéticos (entre 2 y $13 \mathrm{pmol} / \mathrm{l})$ a individuos con DM2 (5-17 pmol/l) y obesidad $^{73,74}$; sin embargo, estos incrementos se dan en los estadios iniciales de la DM2, disminuyendo de manera paralela la secreción de insulina cuando la DM2 es avanzada ${ }^{75}$. En el proceso de envejecimiento se ha encontrado que los depósitos del péptido amiloide aumentan con la edad, relacionándose con disminución de la sensibilidad de la glucosa ${ }^{76}$, ya que la formación de depósitos de amiloide se asocia in vivo a una reducción del 40 al $50 \%$ de la reducción en la función celular en la DM2 ${ }^{77}$. Esta disminución de la sensibilidad está relacionada con la secreción del péptido, la cual se produce de forma paralela a los cambios en la secreción de insulina, indicando que los cambios en este cociente molar amilina/insulina podrían estar involucrados en el desarrollo de la DM2 ${ }^{76-78}$.

La toxicidad de la amilina se da por la inducción apoptótica de las células $\beta$ al formar canales iónicos, principalmente permeables para el calcio, generando un flujo intracelular, donde el incremento de la producción de amilina por estimulación exógena contribuye a la apoptosis ${ }^{79,80}$. Otra teoría indica que la apoptosis se generaría por un aumento en la expresión de los genes promotores de apoptosis $p 53$ y $p 21^{81}$, por lo que se sugiere que los tratamientos con sulfonilureas elevan las concentraciones posprandiales de amilina y que este incremento es el causante de generar depósitos de fibras amiloides y del deterioro de la función celular ${ }^{82}$, algo que no sucede con tratamientos que disminuyen la producción de glucosa hepática ni con insulina o dieta ${ }^{83,84}$.

\section{Consideraciones para los especialistas médicos y salubristas}

La DM2 sigue siendo un problema de salud en todo el mundo y sobre todo en México. Su naturaleza es compleja, dados los múltiples mecanismos moleculares desencadenantes (más de 50 vías implicadas), por lo que el especialista en salud no solo debe comprender la naturaleza poligénica de la DM2, sino que también debe explorar los múltiples factores de riesgo ambientales, ambos con un efecto aditivo, aumentando el riesgo o la predisposición para la diabetes, así como sus complicaciones crónicas, para así poder establecer medidas preventivas y predictivas de esta enfermedad en grupos vulnerables de alto riesgo.

Por ello, se requieren estudios epidemiológicos para establecer el porcentaje de enfermedad que es determinada por los genes y por el ambiente. La interacción de factores también es complicada, y por ello el análisis de todas las teorías e hipótesis existentes sobre la etiología de la DM2 permite una mayor aproximación a la génesis de la enfermedad.

Sin embargo, no se pueden aplicar las múltiples teorías en todas las poblaciones debido a la varianza en cada grupo étnico. Hacen falta más estudios para estimar cuánto contribuyen al riesgo de desarrollo de la enfermedad, siendo el reto que enfrenta la salud desde la epidemiologia molecular en la era posgenómica.

\section{Bibliografía}

1. Cases A. Enfermedad macro y microvascular en la diabetes mellitus tipo 2. Nefrologia (Madr). 2002;13:406-11.

2. Trout GO. Epidemiología de la diabetes mellitus y enfermedad cardiovascular. Duzary. 2007:4:84-94.

3. Organización Mundial de la Salud, Federación Internacional de Diabetes. ¡Actuemos ya! contra la diabetes. Organización Mundial de la Salud (OMS), Federación Internacional de Diabetes; 2003. p. 1-19.

4. Organización Panamericana de la Salud. La diabetes en las Américas. Organización Panamericana de la Salud. Boletín epidemiológico. 2011;22:1-2. 
5. Alvarado C, Milian SF, Valles SV. Prevalencia de diabetes mellitus e hiperlipidemias en indígenas otomíes. Salud Pública Mex. 2001;43:459-63.

6. Carrasco E, Pérez F, Ángel B, et al. Prevalencia de diabetes tipo 2 y obesidad en dos poblaciones aborígenes de Chile en ambiente urbano. Rev Méd Chile. 2004:132:1189-97.

7. Flores LJ, Ramírez SA, Durán P, et al. Molecular heterogeneity of type 2 diabetes mellitus in Mexican Population and its impact of the public health on policies in primary care. Med Chem. 2014:4:791.

8. Ramírez SA, Cabrera CE, Huacuja L, et al. Implicaciones en la atención primaria en salud de la genética y genómica en la diabetes mellitus tipo 2. Rev Med Inst Mex Seguro Soc. 2013;51:e6-26.

9. López F. Diabetes En: López F, editor. Epidemiología: enfermedades transmisibles y crónico degenerativas. México, D.F.: Manual Moderno; 2004. p. 307-24.

10. Masa OJ. Endocrinología molecular: diagnóstico molecular. En: Masa OJ, editor. Diagnóstico molecular. En: Medicina. México, D.F.: Manual Moderno; 2004. p. 198-208.

11. Ariza AR, Nazar RN. Diabetes mellitus y nutrición. En: Casanueva E, Kaufer-Horwitz M, Pérez AB, et al., editores. Nutriología médica. México, D.F.: Médica Panamericana; 2004. p. 371-84.

12. Gungor N, Hannon T, Libman I, et al. DM2 en jóvenes: el cuadro completo hasta la fecha. Pediatr Clin N Am. 2005;52:1579-609.

13. Libman I. Epidemiología de la diabetes mellitus en la infancia y adolescencia: tipo 1, tipo 2 y ¿diabetes "doble"? Rev Argent Endocrinol Metab. 2009:46:22-36

14. Landeros EA. El panorama epidemiológico de la diabetes mellitus. Revista Mexicana de Enfermería Cardiológica. 2000;8:56-9.

15. López $M$, Carmona $A$. La transición alimentaria y nutricional: un reto en el siglo XXI. An Venez Nutr. 2005;18:90-104.

16. Ariza AR, Álvarez CT. Prescripción de agentes orales a pacientes con diabetes mellitus tipo 2. Guía de tratamiento. Rev Med Int Mex. 2008 24:52-8.

17. Secretaria de Salud. Norma Oficial Mexicana, NOM-015-SSA2-2010, para la prevención, tratamiento y control de la diabetes mellitus. Diario Oficial de la Federación. México: Secretaria de Salud; 2001.

18. Rosales RC, López JJ, Núñez NY, et al. Type 2 diabetes nephropathy: a thresholds complex trait and chromosomal morbid map. Rev Med Inst Mex Seguro Soc. 2010;48:521-30.

19. Carrillo C, Panduro A. Genética de la diabetes mellitus tipo 2. Invest Salud. 2001;3:27-34.

20. Neel JV. Diabetes mellitus: a "thrifty" genotype rendered detrimental by "progress"? Am J Hum Genet. 1962;14:353-62.

21. Flores LJ, Dávalos NO, García D, et al. El polimorfismo (CAG)n del gen atxn2, nuevo marcador de susceptibilidad para diabetes mellitus tipo 2. Rev Panam Salud Publica. 2016;40:317-23.

22. Ramírez SA, Carrillo $C$. Detección molecular de una variante en la secuencia del gen hpse que codifica para el dominio del sitio activo de heparanasa y el desarrollo de insuficiencia renal en pacientes con diabetes mellitus tipo 2. Bioquimia. 2009:4:58.

23. Carrillo C, González M, Ramírez SA. Detección molecular de una variante de secuencia del gen que codifica para megalina y el desarrollo de insuficiencia renal causada por diabetes mellitus tipo 2. Bioquimia. 2009;34:59.

24. Topete LR, Ramírez SA, Charles C, et al. Polymorphism g.37190613 $\mathrm{G}>\mathrm{A}$ of the ELMO1 gene in the Mexican population: potential marker for clinical-surgical pathology. Cir Cir. 2014:82:402-11.

25. Valenzuela BA. Why we eat what we eat? Rev Chil Nutr. 2011;38:198-209.

26. Konrad H, German P. Nutrición. Texto y atlas. México D.F.: Médica Panamericana; 2005. p. 342-7.

27. Schnell M, Domínguez ZA, Carrera $C$. Aspectos genéticos, clínicos y fisiopatológicos del síndrome metabólico. An Venez Nutr. 2007;20:92-8.

28. Chacín M, Rojas J, Pineda C, et al. Predisposición humana a la obesidad, síndrome metabólico y diabetes: el genotipo ahorrador y la incorporación de los diabetogenes al genoma humano desde la antropología biológica. Síndrome Cardiometabólico. 2011;1:11-25.

29. Barker DJP. Fetal and infant origins of disease. Eur J Clin Invest. 1995;25:457-63.

30. Lucas A. Programming by early nutrition in man. En: Bock GR, Whelan J, editores. The childhood environment and adult disease. CIBA Foundation Symposium 156. Chichester: John Wiley and Sons; 1991. p. 38-55.

31. Barker DJ, Bull AR, Osmond C. Fetal and placental size and risk of hypertension in adult life. BMJ. 1990;301:259-63.

32. First World Congress Fetal Origins of Adult disease. Pediatr Res. 2001;50:1-66.

33. Cordero P, Milagro FI, Campion J, et al. Epigenética nutricional: una pieza clave en el rompecabezas de la obesidad. Rev Esp Obes. 2010;8:10-20.

34. Fleisch AF, Wright RO, Baccarelli AA. Environmental epigenetics: a role in endocrine disease? J Mol Endocrinol. 2012;49:61-7.

35. Stocker CJ, Arch JR, Cawthorne MA. Fetal origins of insulin resistance and obesity. Proc Nutr Soc. 2005;64:143-51.

36. Bjornsson HT, Sigurdsson MI, Fallin MD, et al. Intra-individual changeover time in ADN methylationwith family alclustering. JAMA. 2008;299:2877-83.
37. Martínez JA, Cordero P, Campión J, et al. Interplay of early-life nutritional programming on obesity, inflammation and epigenetic outcomes. Proc Nutr Soc. 2012;71:276-83.

38. Wren JD, Garner HR. Data-mining analysis suggests an epigenetic pathogenesis for type 2 diabetes. J Biomed Biotechnol. 2005;2:104-12.

39. Milagro FI, Mansego ML, De Miguel C, et al. Dietary factors, epigenetic modifications and obesity outcomes: progresses and perspectives. Mol Aspects Med. 2013;34:782-812.

40. Keating ST, El-Osta A. Chromatin modifications associated with diabetes. J Cardiovasc Transl Res. 2012;5:399-412.

41. Begum G, Stevens A, Smith EB, et al. Epigenetic changes in fetal hypothalamic energy regulating pathways are associated with maternal under nutrition and twinning. FASEB J. 2012;26:1694-703.

42. Ribel R, Fraga MF, Jacobsen S, et al. Genome-wide analysis of DNA methylation differences in muscle and fat from monozygotic twins discordant for type 2 diabetes. PLoS One. 2012;7:e51302.

43. Volkmar M, Dedeurwaerder S, Cunha DA, et al. DNA methylation profiling identifies epigenetic dysregulation in pancreatitis lets from type 2 diabetic patients. EMBO J. 2012:31:1405-26.

44. Madrigal-Ruiz P, Dávalos-Rodríguez NO, Ramírez-García SA, et al. Polymorphism p.D1134A of the TJP1 in population with Zapotec ancestry: a marker for potential thyroid cancer and other neoplasms. Revista Médica MD. 2015;7:20-6.

45. Kyndt T, Quispea D, Zhaic H, et al. The genome of cultivated sweet potato contains Agrobacterium T-DNAs with expressed genes: an example of a naturally transgenic food crop. Proc Natl Acad Sci USA 2015;12:5844-9

46. Seralini GE, Clair E, Mesnage R, et al. RETRACTED: long term toxicity of a Roundup herbicide and a Roundup-tolerant genetically modified maize. Food Chem Toxicol. 2012;50:4221-31.

47. Baschetti R. Genetically unknown foods or thrifty genes? Am J Clin Nutr. 1999:70:420-5.

48. Stubbs RJ, Johnstone AM, O'Reilly LM, et al. The effect of covertly manipulating the energy density of mixed diets on ad libitum food intake in 'pseudo free-living' humans. Int J Obes Relat Metab Disord. 1998:22:980-7.

49. Castro CJ, Villa N, Ramírez SA, et al. Uso medicinal de plantas antidiabéticas en el legado etnobotánico oaxaqueño. Revista Cubana de Plantas Medicinales. 2014;19:101-20.

50. Rafael H. Etiología y fisiopatología de la diabetes mellitus tipo 2. Revista Mexicana de Cardiología. 2011;22:39-43.

51. Godínez SA, Valerdi L. Obesidad: resistencia endocrina múltiple. Rev Endocr Nutr. 2012;20:152-68.

52. Corkey BE. Banting lecture 2011. Hyperinsulinemia: cause or consequence? Diabetes. 2012;61:13-4.

53. Hayden MR, Tyagi SC, Kerklo MM, et al. Type 2 diabetes mellitus as a conformational disease. JOP. 2005;6:287-302.

54. Hayflick M. The serial cultivation of human diploid cell strains. Exp Cell Res. 1961;25:585-892.

55. López JM, Rodríguez D. Mecanismos de envejecimiento celular. Nefrologia (Madr.).1997;17(Supl 3):15-7.

56. Ramírez SA, Flores LJ, Topete LR, et al. High frequency of ancestral allele of the TJP1 polymorphism rs2291166 in Mexican population, conformational effect and applications in surgery and medicine. Cir Cir. 2016;84:28-36.

57. Gershman, R, Gilbert, DL, Nye SW, et al. Oxygen poisoning and x-irradiation: a mechanism in common. Science. 1954;119:623-6.

58. Strehler BL. Ageing: concepts and theories. En: Viidik A, editor. Lectures on gerontology. Academic Press, University of Michigan; 1982. p. 1-7.

59. Quiles JL, Ochoa JJ, Huertas JR, et al. Aspectos mitocondriales del envejecimiento. Papel del tipo de grasa de la dieta y el estrés oxidativo. Endocrinol Nutr. 2004;5:107-20.

60. Cerami A. Hypothesis: glucose as mediator of aging. J Am Geriatr Soc. 1985;33:626-32.

61. Kohn RR, Schnider SL. Glycosylation of human collagen. Diabetes. 1981;31(Supl):47-52.

62. Gems D, Partridge L. Genetics of longevity in model organisms: debates and paradigm shifts. Annu Rev Physiol. 2013;75:621-44.

63. Doonan R, Mc Elwee JJ, Matthi F, et al. Against the oxidative damage theory of aging: superoxide dismutases protect against oxidative stress but have little or no effect on life span in Caenorhabditis elegans. Genes Dev. 2008;22:3236-41.

64. Flores LJ, Ramírez SA, Núñez NY. The metabolic and molecular bases of Cockayne syndrome. Rev Invest Clin. 2010;62:480-90.

65. Slagboom PE, Vug J. Genetic instability and aging: theories, facts, and future perspectives. Genome. 1989:31:373-85.

66. Mozzhukhina TG, Chabanny EL, Levitzky EL, et al. Age-related changes of supranucleosomal structures and DNA-synthesizing properties of rat liver chromatin. Gerontology. 1991;37:181-6.

67. Miquel J, Fleming JE. A two-step hypothesis on them mechanism of in vitro cell aging cell differentiation followed by intrinsic mitochondrial mutagenesis. Exp Gerontol. 1984:19:31-6.

68. Fleming JE, Miquel J, Cottrell SF, et al. Is cell aging caused by respiration dependent injury to the mitochondrial genome? Gerontology. 1982;28:44-53. 
69. Croteau DL, Stierum RH, Bohr VA. Mitochondrial DNA repair pathways. Mutation Research/DNA Repair. 1999;434:137-48.

70. Bohr VA, Anson RM. Mitochondrial DNA repair pathways. J Bioenerg Biomembr. 1999;31:391-8.

71. Sheldrake AR. The ageing, growth, and death of cells. Nature. 1974; 250:381-5.

72. Rojas I, Novials A. Amilina: del estudio molecular a las acciones fisiológicas. Rev Endocrinol Nutr. 2001:48:234-45.

73. Hartter E, Svoboda T, Ludvik B, et al. Basal and stimulated plasma levels of pancreatic amylin indicate its co-secretion with insulin in humans. Diabetologia. 1991;34:52-4.

74. Enoki S, Mitsukawa T, Takemura J, et al. Plasma islet amyloid polypeptide levels in obesity, impaired glucose tolerance and non-insulin-dependent diabetes mellitus. Diabetes Res Clin Pract. 1992;15:97-102.

75. Kahn SE, Verchere CB, Andrikopoulos S, et al. Reduced amylin release is a characteristic of impaired glucose tolerance and type 2 diabetes in Japanese Americans. Diabetes. 1998;47:640-5.

76. Stridsberg M, Sandler S, Wilander E. Cosecretion of islet amyloid polypeptide (IAPP) and insulin from isolated rat pancreatic islets following stimulation or inhibition of $\beta$ cell function. Regul Pept. 1993; 45:363-70.
77. Röcken C, Linke RP, Saeger W. Immunohistology of islet amyloid polypeptide in diabetes mellitus: semi-quantitative studies in a postmortem series. Virchows Arch A Pathol Anat Histopathol. 1992;421:339-44.

78. Novials A, Sarri $Y$, Casamitjana R, et al. Regulation of islet amyloid polypeptide in human pancreatic islets. Diabetes. 1993:42:1514-9.

79. Mirzabekov TA, Lin MC, Kagan B. Pore formation by the cytotoxic islet amyloid peptide amylin. J Biol Chem. 1996;271:1988-92.

80. Bai JZ, Saafi EL, Zhang S, et al. Role of $\mathrm{Ca} 2+$ in apoptosis evoked by human amylin in pancreatic islet $\beta$-cells. Biochem J. 1999;343:53-61.

81. Zhang S, Liu J, Saafi EL, et al. Induction of apoptosis by human amylin in RINm5F islet beta-cells is associated with enhanced expression of p53 and p21WAF1/C1P1. FEBS Lett. 1999;455:315-20.

82. Rachman J, Payne MJ, Levy JC, et al. Changes in amylin and amylin-like peptide concentrations and $\beta$-cell function in response to sulfonylurea or insulin therapy in NIDDM. Diabetes Care. 1998:21:810-6.

83. Lindström $T$, Leckström $A$, Westermark $P$, et al. Effect of insulin treatment on circulating islet amyloid polypeptide in patients with NIDDM. Diabet Med. 1997; 14:472-6.

84. Zapecka B, Czyzyk A, Dworak A, et al. Treatment with metformin in NIDDM patient's lowers plasma amylin level. Diabetologia. 1997; 40(Supl 1):A316. 